\title{
Schwerer Verlust für die Deutsch- Japanische Zusammenarbeit in der Krankenversicherung
}

\author{
Prof. Dr. von Maydell
}

Am 29. April 2006 ist im Alter von 75 Jahren der ehemalige Vize-Präsident des Verbandes der japanischen Betrieblichen Krankenversicherung (Kemporen) Takeshi Shimomura in Tokio verstorben. Herr Schimomura hat nicht nur die Entwicklung der japanischen Krankenversicherung in den letzten Jahrzehnten entscheidend mitgestaltet, er hat auch den Gedankenaustausch zwischen der japanischen und der deutschen Krankenversicherung vorangetrieben und geprägt.

Seine berufliche Karriere im öffentlichen Dienst bis zur Verabschiedung in den Ruhestand im Jahr 1989 legte Takeshi Shimomura im Wesentlichen im Ministerium für Gesundheit und Soziales in Tokio zurück. In dem Ministerium betreute und gestaltete er als Unterabteilungsleiter (seit 1981) die Krankenversicherungreform des Jahres 1984. Auch als Abteilungsleiter (seit 1986) und später als Vize-Präsident von Kemporen (seit 1994) stand die Weiterentwicklung der Krankenversicherung im Mittelpunkt der Arbeit von Takehi Shimomura. Einem ausländischen Beobachter steht es nicht zu, die Bedeutung dieser Arbeit für den japanischen Sozialstaat zu würdigen. Dazu sind die japanischen Fachkollegen berufen.

Wohl aber soll in diesem Beitrag an die Verdienste erinnert werden, die Herr Shimomura sich um die deutsch-japanische Kooperation im Bereich der Gesundheitspolitik erworben hat.

Es war Takeshi Shimomura, der seit den 90er Jahren des vorigen Jahrhunderts immer wieder Europa besuchte, um die dortigen Lösungen für die drängenden Probleme der Krankenversicherung zu studieren. Zu diesem Zweck hat er einen Kooperationsvertrag zwischen Kemporen und dem Max-Planck-Institut für ausländisches und internationales Sozialrecht in München abgeschlossen; im Rahmen dieses Vertrages fanden mehrere Kolloquien und Seminare in Japan und in Deutschland statt, die in mehreren Veröffentlichungen dokumentiert worden sind. ${ }^{1}$ Es ging bei diesem Erfahrungsaustausch, in denen neben der Krankenversicherung auch die Pflegeversicherung einbezogen wurde, nicht nur um die Diskussion der generellen Probleme, wie sie etwa durch die demo- graphischen Herausforderungen geschaffen werden, sondern auch um sehr konkrete Lösungen, wie etwa die DRGs ${ }^{2}$. Beteiligte an diesem intensiven Gedankenaustausch waren auf beiden Seiten Praktiker und Wissenschaftler unterschiedlicher Disziplinen (Ökonomen, Juristen, Mediziner, Sozialwissenschaftler), so dass zu den multinationalen das interdisziplinäre Element hinzukam.

Im Mittelpunkt des vergleichenden Interesses von Takeshi Shimomura stand sicherlich Deutschland, daneben bezog er jedoch auch andere europäische Staaten in seine Untersuchungen ein. So ist auch seine Anregung und mit maßgeblicher finanzieller Unterstützung von Kemporen die vergleichende Studie über die Krankenversicherungssysteme in Frankreich, Deutschland Japan und den Niederlanden entstanden ${ }^{3}$.

Eine schwere Krankheit und sein Ausscheiden aus dem Vorstand von Kemporen führten dazu, dass Takeshi Shimomura die fruchtbare Kooperation zwischen Deutschland und Japan seit 2004 nicht weiter begleiten konnte. Dennoch hatte er noch weitere Pläne, wie er diese Arbeit, die ihm bis zuletzt am Herzen lag, fördern könnte. Davon habe ich mich bei einem Besuch bei ihm wenige Wochen vor seinem Tode überzeugen können.

Takeshi Shimomura hat gezeigt, wie ein internationaler Vergleich sozialpolitisch fruchtbare praktiziert und genutzt werden kann. Auch in Deutschland werden seine Freunde und Bekannte ihm ein dankbares Andenken bewahren.

\section{Fußnoten:}

1 Siehe vor allem, was die Veranstaltungen in Deutschland anbelangt, v. Maydell/Shimomura/Tezuka (Hrsg.), Entwicklungen der Systeme sozialer Sicherheit in Japan und Europa, Schriftenreihe für Internationales und Vergleichendes Sozialrecht, Bd. 17, Berlin 2000.

2 Vgl. den Bericht von Höveler, Deutsch-Japanischer Erfahrungsaustausch zur aktuellen Entwicklung der Kranken- und Pflegeversicherung unter besonderer Berücksichtigung der DRGs, in: Japanisch-Deutsches Zentrum Berlin (Hg.), Reform der sozialen Sicherungssysteme in Japan und Deutschland, jdzb dokumentation Bd. 3, 2003, „. $290 \mathrm{ff}$.

3 Vgl. Henke/Scheyögg, Towards sustainable health care systems. Strategies in health finance schemes in France, Germany, Japan and the Netherlands, A comparative study, IVSS Genf, 2004. 